\title{
A planetary eclipse map of CoRoT-2a
}

\section{Comprehensive lightcurve modeling combining rotational-modulation and transits}

\author{
K. F. Huber, S. Czesla, U. Wolter, and J. H. M. M. Schmitt
}

\author{
Hamburger Sternwarte, Universität Hamburg, Gojenbergsweg 112, 21029 Hamburg, Germany \\ e-mail: khuber@hs.uni-hamburg.de
}

Received 10 July 2009 / Accepted 12 September 2009

\section{ABSTRACT}

\begin{abstract}
We analyze the surface structure of the planet host star CoRoT-2a using a consistent model for both the "global" (i.e., rotationally modulated) lightcurve and the transit lightcurves, using data provided by the CoRoT mission. After selecting a time interval covering two stellar rotations and six transits of the planetary companion CoRoT-2b, we have adopted a "strip" model of the surface to reproduce the photometric modulation inside and outside the transits simultaneously. Our reconstructions show that it is possible to achieve appropriate fits for the entire subinterval using a low-resolution surface model with 36 strips. The surface reconstructions indicate that the brightness on the eclipsed section of the stellar surface is $(6 \pm 1) \%$ lower than the average brightness of the remaining surface. This result suggests a concentration of stellar activity in a band around the stellar equator similar to the behavior observed on the Sun.
\end{abstract}

Key words. techniques: photometric - stars: activity - planetary systems - starspots - stars: individual: CoRoT-2a

\section{Introduction}

Astronomers have long been interested in the surface structure of active stars and their evolution; yet, the surfaces of stars other than the Sun can hardly be resolved directly, so that indirect techniques must be used to obtain an image of the surface. One such technique is Doppler imaging (Vogt \& Penrod 1983), which requires a dense series of high-resolution spectra and stellar rotation velocities of $v \sin (i) \gtrsim 20 \mathrm{~km} \mathrm{~s}^{-1}$ (compared to $v_{\mathrm{eq}} \approx 2 \mathrm{~km} \mathrm{~s}^{-1}$ for the Sun). Alternatively, lightcurves also yield information on stellar surface structures and can usually be obtained at low observational cost. However, photometry provides less information and the problem of lightcurve inversion is known to be notoriously ill-posed.

Since the launch of CoRoT in 2006, an increasing amount of high-quality space-based photometry has become available. Without the limitations the atmosphere and the day-night cycle impose on ground-based observatories, CoRoT is able to provide photometry with unprecedented temporal coverage and cadence, which is enormously interesting in the context of stellar activity and surface reconstruction.

In the course of the CoRoT planet-hunting project, the giant planet CoRoT-2b (Alonso et al. 2008) was detected. The host star of this planet, CoRoT-2a, is solar-like in mass and radius, but rotates approximately four times faster than the Sun and is considerably more active. The planet orbits its host star approximately three times per stellar rotation and, during its passage across the stellar disk, acts as a shutter scanning the surface of the star along a well-defined latitudinal band. Because the "local" surface structure is imprinted on the transit profiles (Wolter et al. 2009; Czesla et al. 2009), they can be used to partially resolve the ambiguity of the lightcurve inversion problem.

While Lanza et al. (2009) used the "global" lightcurve of the host star to reconstruct its surface inhomogeneities, without considering the transits, Wolter et al. (2009) concentrated on a single transit lightcurve to reconstruct a fraction of the surface, neglecting the "global" lightcurve. In this work, we combine and refine these approaches to present a reconstruction that simultaneously describes both the overall lightcurve and the transits during two stellar rotations.

\section{Observations and data reduction}

Alonso et al. (2008) discovered the planet CoRoT-2b using photometric data provided by the CoRoT mission (for a detailed description, see Auvergne et al. 2009). The planet was detected in the field observed during the first long run carried out between May 16 and Oct. 15, 2007. The default sampling rate of CoRoT photometry is $1 / 512 \mathrm{~s}^{-1}$. The CoRoT-2 lightcurve was observed at this rate only for the first five days, after which the transits were detected and the satellite switched to alarm mode, continuing to take data every $32 \mathrm{~s}$. The light collected by the CoRoT telescope is dispersed using a prism and recorded by a CCD chip. Individual sources are separated by a photometric mask, which also defines three broadband channels (nominally red, green, and blue). Currently, there is no appropriate calibration available for these channels, so that it is unfeasible to use the color information in this work. The signal obtained by summing up the individual channels, often referred to as "white light", corresponds to an optical measurement with a filter transmission maximum in the red wavelength region (Auvergne et al. 2009). Accordingly, Lanza et al. (2009) assume an isophotal wavelength of $700 \mathrm{~nm}$ for their passband. The CoRoT data undergo a standard pipeline processing, during which data points are flagged that are significantly affected by known events, as for example the South Atlantic Anomaly (SAA), so that they can be removed from the lightcurve. 
Table 1. Stellar/planetary parameters of CoRoT-2a/b.

\begin{tabular}{lcc}
\hline \hline Star $^{a}$ & Value \pm Error & Ref. $^{b}$ \\
\hline$P_{\mathrm{s}}$ & $(4.522 \pm 0.024) \mathrm{d}$ & L09 \\
$P_{\mathrm{s}}^{*}$ & $4.57 \mathrm{~d}$ & \\
Spectral type & $\mathrm{G} 7 \mathrm{~V}$ & B08 \\
\hline \hline Planet $^{c}$ & Value \pm Error & Ref. \\
\hline & & \\
$P_{\mathrm{p}}$ & $(1.7429964 \pm 0.0000017) \mathrm{d}$ & $\mathrm{A} 08$ \\
$T_{\mathrm{c}}[\mathrm{BJD}]$ & $(2454237.53362 \pm 0.00014) \mathrm{d}$ & $\mathrm{A} 08$ \\
$i$ & $(0.172 \pm 0.001)$ & $\mathrm{C} 09$ \\
$R_{\mathrm{p}} / R_{\mathrm{S}}$ & $(6.70 \pm 0.03)$ & $\mathrm{C} 09$ \\
$a / R_{\mathrm{S}}$ & $(0.41 \pm 0.03),(0.06 \pm 0.03)$ & $\mathrm{A} 08$ \\
$u_{a}, u_{b}$ & A08 \\
\hline
\end{tabular}

${ }^{a} P_{\mathrm{s}}-$ stellar rotation period, $P_{\mathrm{s}}^{*}-$ stellar rotation period used for the observation interval analyzed in this paper (see Sect. 3.4). ${ }^{b}$ Taken from Lanza et al. (2009) [L09], Alonso et al. (2008) [A08], Bouchy et al. (2008) [B08], or Czesla et al. (2009) [C09]. ${ }^{c} P_{\mathrm{p}}-$ orbital period, $T_{\mathrm{c}}-$ central time of first transit, $i$ - orbital inclination, $R_{\mathrm{p}}, R_{\mathrm{s}}-$ planetary and stellar radii, $a$ - semi major axis of planetary orbit, $u_{a}, u_{b}-$ linear and quadratic limb darkening coefficients.

The host star CoRoT-2 has a spectral type of G7V with an optical companion at a distance of approximately 4.3" (2MASS, Skrutskie et al. 2006), too close to be resolved by CoRoT. According to Alonso et al. (2008) the secondary contributes a constant fraction of $(5.6 \pm 0.3) \%$ to the total CoRoT-measured flux. In Table 1 we list the system parameters of CoRoT-2a/b, which are used throughout our analysis. CoRoT-2b's orbital period of $\approx 1.74$ days is about a third of CoRoT-2a's rotation period; hence, the almost continuous CoRoT data sample of 142 days covers about 30 stellar rotations and more than 80 transits. The lightcurve shows signatures of strong stellar activity and substantial rotational modulation (Lanza et al. 2009). We use the same CoRoT raw data reduction procedures as described in Czesla et al. (2009, Sect. 2).

\section{Analysis}

\subsection{Modeling approach}

The measurements of the Rossiter-McLaughlin effect by Bouchy et al. (2008) suggest that the rotation axis of the host star and the planet's orbit normal are approximately co-aligned $\left(\lambda=7.4 \pm 4.5^{\circ}\right)$. The sign $\lambda$ represents the misalignment angle projected on the plane of the sky, and its value strongly favors aligned orbital and rotational axes, even though it does not prove it. More support for a co-aligned geometry comes from the following argument. Comparing the measured $v \sin (i)=11.85 \mathrm{~km} \mathrm{~s}^{-1}$ with a calculated equatorial velocity of $v_{\mathrm{eq}}=2 \pi R_{\mathrm{S}} / P_{\mathrm{s}} \approx 10 \mathrm{~km} \mathrm{~s}^{-1}$ derived with the theoretically obtained value $R_{\mathrm{S}}=0.9 \cdot R_{\odot}$ (Alonso et al. 2008) also favors $\sin (i) \approx 1$.

As a result, the planet always eclipses the same low-latitude band between 6 and 26 degrees. The transits separate the stellar surface into two observationally distinct regions, i.e., a region eclipsed by CoRoT-2b and another region that is not. In the case of CoRoT-2a, the eclipsed section covers $\approx 21 \%$ of the stellar disk corresponding to $\approx 17.3 \%$ of its surface. The time-resolved planet migration across the visible stellar disk sequentially covers and uncovers surface fractions, so that the brightness profile of the underlying stellar surface is imprinted on the transit lightcurve.

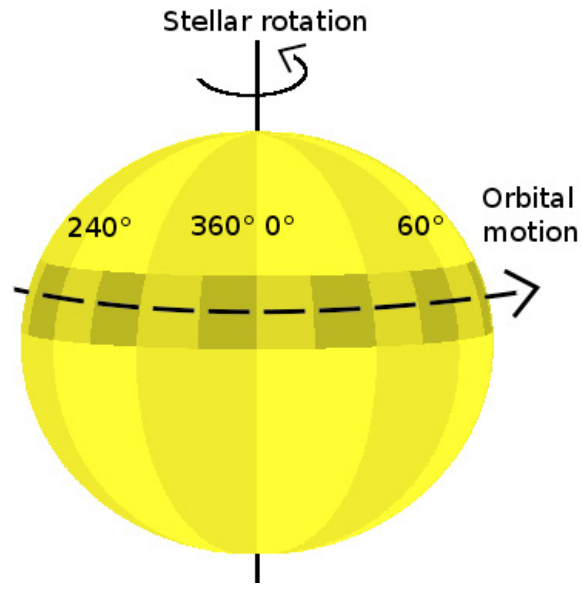

Fig. 1. Our model geometry using 12 longitudinal strips for the noneclipsed and 24 strips for the eclipsed section, respectively.

For our modeling, we separate the surface into the eclipsed and the noneclipsed section, which are both further subdivided into equally sized, longitudinal bins or "strips" as demonstrated in Fig. 1. Let $N_{\mathrm{e}}$ be the number of bins in the eclipsed section and $N_{\mathrm{n}}$ be the number of noneclipsed strips. As is apparent from Fig. $1, N_{\mathrm{e}}$ and $N_{\mathrm{n}}$ need not be the same. Altogether, we have $N_{\text {tot }}=N_{\mathrm{e}}+N_{\mathrm{n}}$ bins enumerated by some index $j$. A brightness $b_{j}$ is assigned to each of these surface bins, with which it contributes to the total (surface) flux of the star. Then let $V_{j i}$ denote the visibility of the $j$ th bin at time $t_{i}$. The visibility is modified in response to both a change in the viewing geometry caused by the stellar rotation and a transit of the planet. The modeled flux $f_{\text {mod, } i}$ at time $t_{i}$ is then given by the expression

$f_{\mathrm{mod}, i}=\sum_{j=1}^{N_{\mathrm{tot}}} V_{j i} b_{j}$.

We determine the unknown brightnesses, $b_{j}$, by comparing $f_{\text {mod }, i}$ to a set of $M_{\mathrm{C}}$ CoRoT flux measurements using a specifically weighted version of the $\chi^{2}$-statistics:

$\chi_{\mathrm{m}}^{2}=\sum_{i=1}^{M_{\mathrm{C}}} \frac{\left(f_{\mathrm{mod}, i}-f_{\mathrm{obs}, i}\right)^{2}}{\sigma_{i}^{2}} \cdot w_{i}$,

where $\chi_{\mathrm{m}}^{2}$ differs from $\chi^{2}$ by a weighting factor, $w_{i}$, which we choose to be 10 for lightcurve points in transits and 1 otherwise. In this way, the global lightcurve and the transits are given about the same priority in the minimization process. Error bars for the individual photometric measurements were estimated from the datapoint distribution in the lightcurve, and the same value of $\sigma=1000 \mathrm{e}^{-} / 32 \mathrm{~s}\left(=1.4 \times 10^{-3}\right.$ after lightcurve normalization $)$ was used for all points.

In our modeling we currently exclude surface structures with a limb-angle dependent contrast. This particularly refers to solar-like faculae, for which Lanza et al. (2009) find no evidence in their analysis. The planet CoRoT-2b is modeled as a dark sphere without any thermal or reflected emission. This approximation is justified by the findings of Alonso et al. (2009), who report a detection of the secondary transit with a depth of $(0.006 \pm 0.002) \%$, which is negligible in our analysis.

The actual fit is carried out using a (nongradient) Nelder-Mead simplex algorithm (e.g. Press et al. 1992). All strips are mutually independent, and as we define only a rather 
coarse strip subdivision for the noneclipsed section of the surface and since the eclipsed section is thoroughly covered by the transits, no further regularization is necessary.

\subsection{Fit parameter space - parametrization, restrictions, and interpretation}

Our fit space has a total of $N_{\text {tot }}=N_{\mathrm{e}}+N_{\mathrm{n}}$ dimensions, and the associated parameters are the brightnesses, $b_{j=1 \ldots N_{\mathrm{tot}}}$. The most obvious choice of fit parameters are the brightnesses themselves. Nevertheless, using a slightly different definition in our algorithm provides some advantages. Instead of using the brightness of the global strips in our fits, we replace them with a weighted sum of the brightnesses of the eclipsed and the noneclipsed strips covering the same longitudes. This quantity $z$ is a measure of the total flux emitted from all strips enclosed within a certain longitude range and, therefore, represents the level of the global lightcurve independent of how the brightness is distributed among the individual strips contributing to the sum. Without any transit observations, the distribution of flux among the individual contributors could hardly be restricted further, because latitudinal information could not be recovered. Thus, we use the tuple $\left(b_{l=1 \ldots N_{\mathrm{e}}}, z_{k=1 \ldots N_{\mathrm{n}}}\right)$ for our reconstructions, where $z$ is defined by

$z_{k}=b_{N_{\mathrm{e}}+k}+\frac{1}{c \cdot q} \sum_{s=q_{0}}^{s<q_{0}+q} b_{\mathrm{s}}$.

In Eq. (3), $b_{N_{\mathrm{e}}+k}$ denotes the brightness of the $k$ th global strip, $q$ is defined by $N_{\mathrm{e}} / N_{\mathrm{n}}$ (the factor by which the eclipsed section is oversampled compared to the noneclipsed section), the index range $q_{0} \leq s<q_{0}+q$ enumerates all eclipsed strips covering the same longitudes as the global strip referred to by $b_{N_{\mathrm{e}}+k}$, and $c$ is a scaling factor accounting for the size difference between the eclipsed and the noneclipsed sections.

The practical advantage of using $z$ instead of the brightness values themselves lies in the parameter interdependence. If we assume a fit algorithm adjusts the structure of a transit lightcurve using the eclipsed strips, every modification of their brightness causes a modification of the global lightcurve level, which might possibly be compensated by an appropriate adjustment of the global strip's brightness. Such an adjustment is inherent in the definition of $z$, so that $b_{l=1 \ldots N_{\mathrm{e}}}$ and $z_{k=1 \ldots N_{\mathrm{n}}}$ become largely independent quantities. In our fits we use $c=5$, which roughly corresponds to the ratio of disk area covered by global and eclipsed strips.

To normalize the observed CoRoT-2a lightcurve, we divided all measurements by the highest flux value in our lightcurve so that $0<$ (normalizedflux) $\leq 1$. The matrix $V_{j i}$ in Eq. (1) is normalized according to

$\sum_{j=1}^{N_{\text {tot }}} V_{j i}=1$ for all $i$,

which yields $f_{\text {mod, } i}=1$ for $b_{j}=1$, i.e., a constant model lightcurve at level 1. In a first, tentative interpretation, a surface element with the brightness 1 corresponds to a photospheric element free of any spots. Nonetheless, this is only correct as long as we assume that the largest observed flux in the lightcurve indeed represents the "spot-cleaned" photospheric luminosity. As CoRoT-2a is, however, a very active star, it seems probable that polar spots persist on its surface. Moreover, it seems likely that lower latitude structures cover a fraction of the stellar surface even if the lightcurve is at maximum. For this reason, individual surface elements (strips) may be brighter than the "average surface" during the maximum observed flux. While such information could not be recovered if no transits were observed, individual surface regions eclipsed by the planet can conceivably be brighter than the "global" photosphere seen during lightcurve maximum. Therefore, we do not exclude strips with brightness values above 1 in our fits; i.e., we do not fix the photospheric brightness. This results in brightnesses above 1 for individual strips (e.g. Fig. 5). The only parameter space restriction applied during our fits is that the brightness must be positive.

\subsection{Which part of the lightcurve should be used?}

To derive a meaningful model, we need to select a time span, which is both long enough to provide an appropriate coverage of the surface, and short enough to minimize the effects of surface evolution. The latter, while doubtlessly present, appears slow compared to the stellar rotation period. Lanza et al. (2009) give typical lifetimes of $55 \mathrm{~d}$ ( $\approx 12$ rotations) for active regions and 20-30 d for some individual spots. In our analysis, we use the time span ranging from phase 1.85 through 3.85 (BJD $=2454245.988$ to $\mathrm{BJD}=2454255.128$, $\mathrm{BJD}=$ Barycentric Julian Date), which covers 6 transits and shows only small variations in the global lightcurve. The data are re-binned using a binsize of $128 \mathrm{~s}$ for the transit covered periods and $2016 \mathrm{~s}$ for the remaining lightcurve.

Our binning approach has to take into account interruptions of the lightcurve due to data drop outs (for instance caused by the South Atlantic Anomaly) and, of course, has to account for the change in bin size when a transit period begins or ends. Moreover, the CoRoT-2 lightcurve is sampled at two different rates $\left(1 / 512 \mathrm{~s}^{-1}\right.$ and $\left.1 / 32 \mathrm{~s}^{-1}\right)$, which does not, however, cause any problem during the time span under consideration here. To obtain the binned curve, we averaged all flux values comprised of a bin and place the resulting value at the barycenter of the contributing time stamps. To compute the error, we divided the standard deviation for individual points by the square root of the number of averaged points. With this approach we (typically) obtain an error of $7 \times 10^{-4}$ for in-transit points and $1.8 \times 10^{-4}$ for out-of-transit points.

In Fig. 2 we demonstrate the coverage of the eclipsed surface section by these 6 transits within the selected phase interval. A single rotation phase including three transits only provides a very inhomogeneous "scan" of the eclipsed surface because of limb darkening, projection geometry, and the distribution of transit intervals (cf. Fig. 2). As a transit occurs every $\approx 0.4$ stellar rotations, a homogeneous coverage of one full rotation is achieved using five transits. Nonetheless, we decided to use an integer number of stellar rotations and used six transits with the last one showing virtually the same part of the eclipsed surface as the first.

\subsection{Surface evolution, rotation period, and model limits}

Although the lightcurve of CoRoT-2a shows remarkably periodic minima and maxima, the rotation period of the star is not known exactly. Using a Lomb-Scargle periodogram, Lanza et al. (2009) find a rotation period of $(4.52 \pm 0.14)$ d for the star, which is further refined in the course of their surface modeling. Assuming that the longitudinal migration of the active longitudes should be minimal, Lanza et al. pin down the stellar rotation period to $4.5221 \mathrm{~d}$. While this rotation period minimizes the 




Fig. 2. Visibility of the eclipsed stellar surface during the transits in the selected observation interval. Low visibility means that a stellar feature at the corresponding longitude has a low impact on the transit profiles.

migration of the active longitudes, it results in individual spots showing a retrograde migration with an apparent angular velocity $\approx 1.3 \%$ lower than the stellar rotation.

For our modeling we determine an "effective" period, representing the rotation period of the dominating surface features we are mainly interested in. We use the selected part of the lightcurve, remove the transits, and fold the remaining lightcurve back at a number of periods between $4.4 \mathrm{~d}$ and $4.7 \mathrm{~d}$. The best match is obtained using a period of $4.57 \mathrm{~d}$, which also results in the best fits of our models, so we use it throughout our analysis. This period also agrees with the values given by Lanza et al. (2009) assuming a rotation period of $4.5221 \mathrm{~d}$ and a mean retrograde migration "slowing down" the spots by $1.06 \%$; however, changes in the rotation period on this scale do not result in significantly different surface reconstructions.

Even though we identified a lightcurve interval with relatively weak surface evolution, and refined the rotation period to account for some evolutionary effects, there is still a remaining modulation. This modulation imposes a fundamental limit on the fit quality that can be achieved by adopting a static model to the lightcurve, because both stellar rotations have to be described by the same model. To estimate this limit, again for the global lightcurve alone, we estimated the quantity

$$
\left\langle\Delta \chi^{2}\right\rangle=\frac{1}{N} \sum_{i=1}^{N}\left(\frac{f\left(p_{i}\right)-f\left(p_{i}+1.0\right)}{2 \sigma}\right)^{2} \approx 14.2 .
$$

Here, $f\left(p_{i}\right)$ is the normalized flux in the $i$ th phase bin, $f\left(p_{i}+1.0\right)$ is the flux measured at the same phase during the next stellar rotation, and the sum stretches over all phases pertaining to the first rotation. The flux $f\left(p_{i}+1.0\right)$ was obtained by interpolation, because the phase sampling is not exactly the same in both rotations. Since $\sigma_{i} \approx \sigma$, the best conceivable common model with respect to $\chi^{2}$ at phase point $p_{i}$ is given by $\left(f\left(p_{i}\right)+f\left(p_{i}+1.0\right)\right) / 2$, so that the sum in Eq. (4) estimates the $\chi^{2}$ contributions induced by surface evolution for each point. If there was no surface evolution, the expression in Eq. (4) would equate to 0.5, because statistical errors are, of course, still present. Therefore, a limit of $\chi^{2} \approx 14.2$ per (global) lightcurve point will not be overcome by any static model. Equivalently, the expectation value, $\langle\Delta f / 2\rangle$, for the flux deviation from the best model equals $\langle\Delta f / 2\rangle=\left\langle\left(f\left(p_{i}\right)-f\left(p_{i}+1.0\right)\right) / 2\right\rangle=5.6 \times 10^{-4}$ and cannot be surpassed.

\subsection{Model resolution}

The parameters $N_{\mathrm{e}}$ and $N_{\mathrm{n}}$ specify the model resolution of the eclipsed and noneclipsed sections. An appropriate choice of these parameters balances fit quality and model ambiguity; this way the largest possible amount of information can be extracted.

To find the optimal value for the number of noneclipsed strips, we carry out fits to only the global lightcurve using an increasing number of global strips. Starting with only 4 strips, we find the reduced $\chi^{2}$ value, $\chi_{\mathrm{R}}^{2}$, to decrease rapidly until 8 strips are used. From this point, $\chi_{\mathrm{R}}^{2}$ only responds weakly to an increase in the strip number, but still decreases. Using 12 strips, we find $\chi_{\mathrm{R}}^{2}=16$. With an estimated "socket" contribution of $\approx 14.4$ provided by surface evolution, we attribute a fraction of $\chi_{\mathrm{R}}^{2} \approx 1.6$ to statistical noise. This fraction decreases to $\approx 1$ if we use 30 strips, in which case we obtain a longitudinal resolution of $12^{\circ}$, comparable to what is achieved by Lanza et al. (2009). According to our test runs, we obtain reasonably stable results using 12 strips. As the stability of the solutions decreases for larger strip numbers, while $\chi_{\mathrm{R}}^{2}$ only slightly improves, we argue in favor of using 12 global strips in our modeling, to extract the largest possible amount of physically relevant results.

The resolution used on the eclipsed surface band is determined according to the following considerations. The extent of the planetary disk at the center of the stellar disk is about $20^{\circ} \times 20^{\circ}$. All stellar surface elements simultaneously (un)covered by the planet's disk are equivalent in our lightcurve modeling. Individual features can, thus, be located (or smeared out) along the edge of the planetary disk to provide the same effect in the lightcurve. This edge stretches across $10^{\circ}$ in longitude (only the "forward" part) and $20^{\circ}$ in latitude, which defines a fundamental limit for the resolution. Assuming a particular shape for the features, decreases the degree of ambiguity as was for example shown by Wolter et al. (2009).

A meaningful structure in the transit profile should comprise at least 3 consecutive lightcurve bins corresponding to about $360 \mathrm{~s}$ or $\approx 6^{\circ}$ of planet movement across the center of the stellar disk. The extent of individual strips should, therefore, not fall below this limit, but be even larger.

Combining these arguments with the results of our test runs, we decided to use 24 strips on the eclipsed section, so that a longitudinal resolution of $15^{\circ}$ is achieved. With this choice, a single strip on the eclipsed band appears about the same size (face-on) as the planetary disk. Additionally, we note that this approximately corresponds to the resolution used by Lanza et al. (2009) in their maximum entropy reconstructions.

\subsection{Results of the modeling}

In our analysis, we achieve a longitudinal resolution of $\approx 15^{\circ}$ on the eclipsed section making up $\approx 17 \%$ of the stellar surface and $30^{\circ}$ for the rest.

In Figs. 3 and 4 we present the results of our modeling. Figure 3 shows the entire subsample of CoRoT data points used in our modeling, along with our lightcurve model in the upper panel. In the lower panel we show the model residuals (see Sect. 3 for the definition of the error). Obviously, the data are matched well; however, there are systematic offsets between the observation and the model. In particular, the model tends to overestimate the observations during the first half of the time span, whereas it underpredicts it in the second half. This effect is related to surface evolution already detectable on timescales below the rotation period (Lanza et al. 2009, also see Sect. 3.4). Within the transits the residuals remain small compared to the rest of the lightcurve. This must be regarded a consequence of both the smaller bin size of $128 \mathrm{~s}$ used here and the twice better resolution of the model on the eclipsed section. During the fit the 


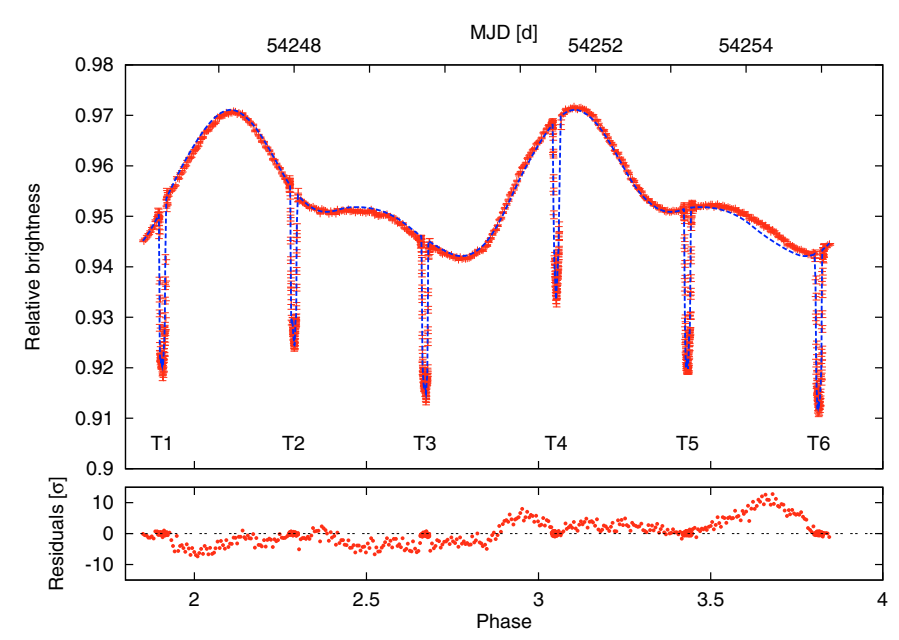

Fig. 3. Upper panel: CoRoT data for rotational phases 1.85 to 3.85 (2016 s binning for the global lightcurve and $128 \mathrm{~s}$ for the transits, red symbols) and our model lightcurve (dashed blue curve). Lower panel: residuals of our model.

transit residuals are "overweighted" by a factor of ten to avoid them to be prevailed by the much larger global residuals. Although, the deviations can be as large as $10 \sigma$, the mean deviation of the global lightcurve from the model amounts to $620 \times 10^{-6}$ not far from the theoretical limit of $560 \times 10^{-6}$ (cf. Sect. 3.4).

The lightcurve presented in Fig. 3 contains six transits (labeled "T1 -6 "). The associated transit lightcurves are shown in detail with our models in Fig. 4. Each individual panel shows the same transit twice: the lower curve represents a transit reconstruction from the full data sample (phases 1.85-3.85), and the upper curve denotes a reconstruction from only the first $(T 1-3)$ or second (T4-6) half of the sample data (shifted up by 0.03 ). The dotted lines show the transits as we would observe them without any activity on the eclipsed section of the surface, where we assume a brightness of 1 for the underlying photosphere.

The transit reconstructions obtained from half of the sample data reproduce the transit substructure very accurately. The resulting surface reconstructions are, however, unreliable where the surface is insufficiently covered (cf. Fig. 2, around longitudes of $180^{\circ}$ and $320^{\circ}$ ). Interestingly, those reconstructions based on data from two rotation phases also recover most of the transit substructure and are by no means off the mark. When both rotation periods are used, $\chi^{2}$ typically increases by $10-20 \%$, a difference hardly visible in Fig. 4. As an exception, the fit quality of the third transit (T3) decreases dramatically, with $\chi^{2}$ increasing by a factor of $\approx 2.5$. This is, however, mainly a consequence of the observed surface evolution shifting the continuum level. The overall stability of the fit quality indicates that lifetimes of surface features are a few stellar rotations, which agrees with the results of Lanza et al. (2009).

In Fig. 5 (lower and middle panels) we present the strip brightness distribution pertaining to the lightcurve model shown in Figs. 3 and 4, i.e., a 1D-reconstruction of the surface. We estimated mean and errors by recording the distribution of the parameter values obtained from 50 reconstructions with randomized starting points, and the respective distributions are indicated by the color gradients in Fig. 5. The error bars correspond to the associated standard deviations. They reflect the ability of the fitting algorithm to converge to a unique extremum, which is determined by both the characteristics of the algorithm and the structure of the fit statistics. Investigating the brightness
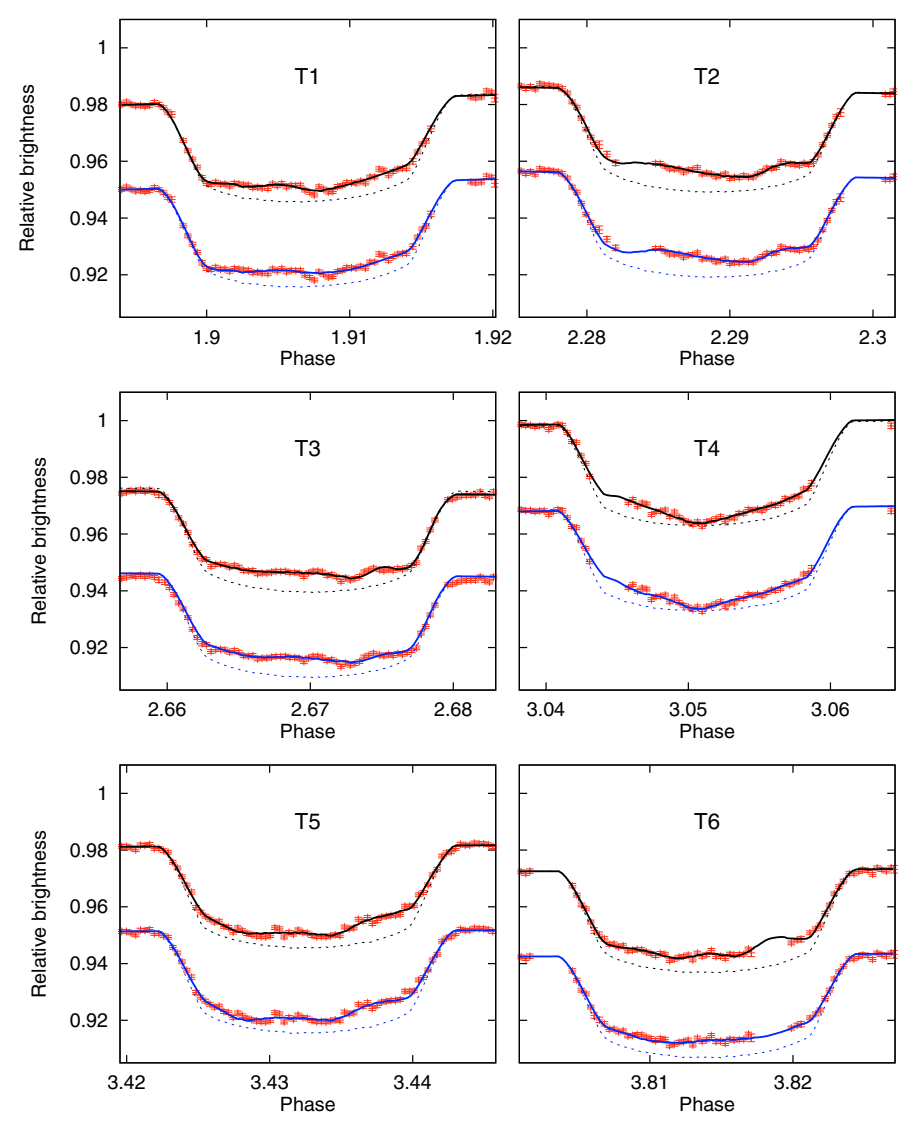

Fig. 4. Close-up of the six individual transits (128 s binning). Observations are drawn as red points (including $1 \sigma$ errors). The upper curve (black) in each panel shows the fit obtained from 1 rotation (phases 1.85 to 2.85 for $T 1-3$ and 2.85 to 3.85 for $T 4-6$ ); the lower curve (blue) gives the fit obtained by modeling both rotations (as seen in Fig. 3). The dashed lines show the undisturbed transit profile for comparison.

distribution of the noneclipsed strips, we notice a slight degeneracy in some of the 12 noneclipsed strips; i.e., a fraction of the brightness may be redistributed without considerable loss of fit quality. The averaging of the 50 reconstructions flattens out such features, thus acting like a regularization of the brightness distribution. No such effect is observed for the eclipsed strips.

We compared our results to the reconstructions given by Lanza et al. (2009, their Fig. 4) and find our longitude scale to be shifted by $\approx 70^{\circ}$ with respect to the Lanza et al. scale. Our reconstructions show the same bright band at a longitude of $\approx 260^{\circ}$ ( $330^{\circ}$ in our work). Tentatively averaging over an appropriate "time band" in their Fig. 4, we also find qualitative agreement for the remaining spot distribution.

Clearly, the flux fraction contributed by the eclipsed strips is less than that of the noneclipsed strips, because the area they cover is smaller by a factor of five. In the upper panel of Fig. 5 we show the lightcurve model contributions provided by the eclipsed and the noneclipsed section with their sum making up the model for the CoRoT data, which is also shown. The median flux level was subtracted from all curves to emphasize the modulation amplitude in favor of flux level. Obviously, the modulation amplitudes induced by the eclipsed and noneclipsed section balance approximately. This indicates that their influence on the stellar variability is about the same despite their large difference in size. 

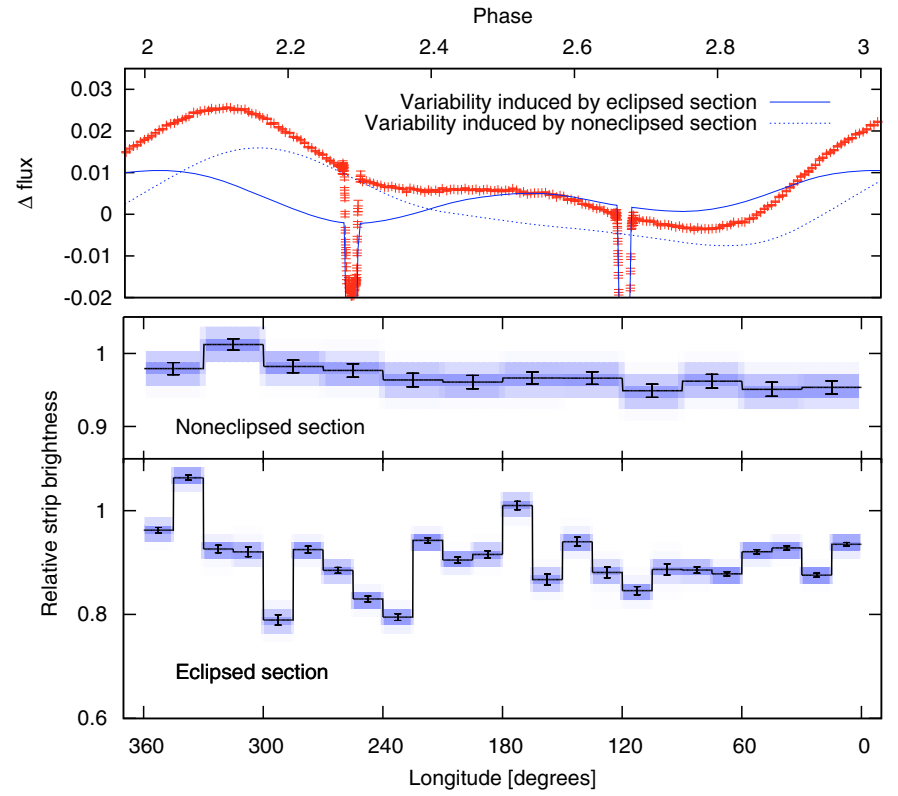

Fig. 5. Upper panel: the CoRoT data (red symbols) and the flux modulation contributed in our model by the eclipsed (blue curve) and noneclipsed section (dotted gray curve) individually. All curves are median-subtracted. Middle and lower panels: Brightness distribution of the strips located on the eclipsed and noneclipsed sections of the surface. The color gradient renders the distribution obtained from 50 reconstructions with randomized starting points.

A visualization of our best-fit surface reconstruction is presented in Fig. 6, showing a Hammer projection of the associated distribution of surface brightnesses. The planet-defined lowlatitude band shows especially dark features, e.g. in the range of $200^{\circ}$ to $300^{\circ}$ in longitude, and is clearly visible. Also the noneclipsed sections of the star show significant variations. Note that our map only shows the average brightness of these regions; since the noneclipsed regions are larger by area, they contribute more flux; however, the "missing" flux in these regions is likely to also be concentrated in spots. In the following section we address the issue of the flux contribution from the eclipsed and noneclipsed sections.

\subsubsection{Brightness distribution and spot coverage}

Without a very precise absolute flux calibration (as e.g. in Jeffers et al. 2006), lightcurve analyses can usually only investigate the inhomogeneous part of the entire spot coverage. This statement is, however, partially invalidated by a transiting planet because it breaks the symmetry of the problem. Spots eclipsed by the planetary disk distort the transit profiles regardless of whether they belong to a structure that appears symmetric on a global scale or not.

As an example, assume that half the eclipsed section of CoRoT-2a, say longitudes $0-180^{\circ}$, is spotted, while the other half is covered by an undisturbed photosphere. Clearly, the transits will be shallower when the planet eclipses the dark portion of the star, and they will be deeper when the bright section is eclipsed. Also, the lightcurve will be distorted. Assume as well that a comparable section between longitudes $180-360^{\circ}$ is dark on the opposite hemisphere of the star outside the eclipsed band. In this case the global spot configuration is perfectly symmetric with respect to longitude and the global lightcurve does not show any trace of activity. Nevertheless, the transits will still be

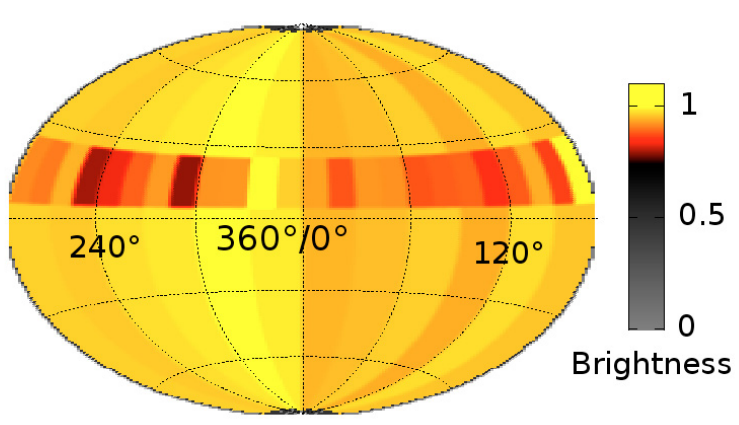

Fig. 6. Surface map of CoRoT-2a showing the reconstructed brightness distribution. Spots located on the noneclipsed surface are blurred over the entire reconstruction strip because of their unknown latitude resulting in the lower contrast compared to the eclipsed section.

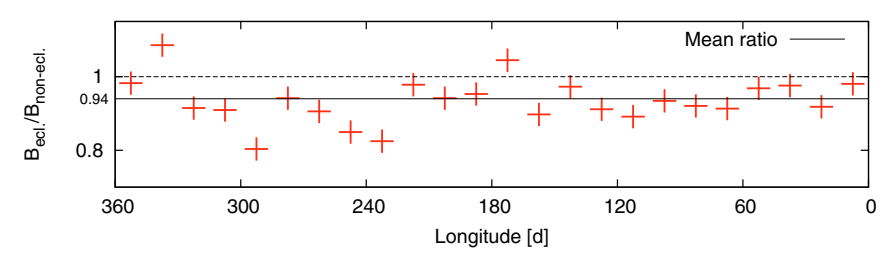

Fig. 7. Ratio of the surface brightness in the eclipsed $\left(B_{\text {ecl. }}\right)$ and noneclipsed ( $\left.B_{\text {non-ecl. }}\right)$ sections.

shallower when the planet eclipses the dark band, and they will still be deeper when the bright surface is eclipsed.

If the spots were distributed symmetrically across the stellar surface, we would expect the stellar surface to be homogeneously bright. In Fig. 7 we show the brightness ratio of eclipsed and noneclipsed sections as a function of longitude. Since there are more eclipsed than noneclipsed strips, we always compare strips covering the same longitude. Only in two cases is the eclipsed section brighter than its noneclipsed counterpart, while in 22 cases it is not.

The mean ratio is $0.94 \pm 0.01$ so that the part of the star passingly covered by the planet is found to be $6 \%$ darker than the rest of the surface. The remaining (nontransited) surface is brighter on average, but locally it may even be darker.

\section{Discussion and conclusions}

We present a surface reconstruction for the planet host star CoRoT-2a. Our modeling is based on a CoRoT data interval covering two full stellar rotations, and it treats the entire lightcurveincluding the planetary transits - in a consistent way.

We show that a consistent modeling of the lightcurve is possible using a static model, i.e., not including any spot evolution. Although surface evolution on scales of the stellar rotation period is seen in both the reconstruction of the global lightcurve (as already reported by Lanza et al. 2009) and the transit lightcurves, this effect is weak in the context of our analysis. The static model provides reasonable fits to six consecutive transit lightcurves. The associated surface configuration changes little during this period, so the surface evolution must be relatively slow compared to the timescale of $\approx 9 \mathrm{~d}$ under consideration. This timescale is also valid for the lifetimes of spots on the eclipsed surface section.

Our results indicate that the planet-eclipsed band on the stellar surface is - on average - about $6 \%$ darker than the remaining part of the surface. Lanza et al. (2009) note that the strength 
of differential rotation derived from their lightcurve fits seems much lower than the expected values derived from measurements in other systems (Barnes et al. 2005). They speculate that this may indicate a spot distribution limited to a narrow latitude band. If this is true, the latitude band is possibly located at low latitudes, i.e., within $\pm 30^{\circ}$ around the equator as observed on the Sun. In this case it covers the eclipsed section where we find a darker surface, i.e., higher spot coverage. We caution that this result may also be influenced by the adopted planetary parameters (mainly the size), which are hard to determine accurately (Czesla et al. 2009).

We checked whether the effect of gravitational darkening could significantly contribute to a darker surface in the vicinity of the equator. For the stellar parameters of CoRoT-2a, we find that the (effective) gravitational accelerations at the poles and at the equator are equal to within $0.07 \%$, so that gravitational darkening does not significantly contribute to the brightness gradient found in our modeling. This result is nearly independent of the assumed coefficient, $\beta_{1}\left(T_{\text {eff }}^{4} \propto g^{\beta_{1}}\right.$ with the effective surface temperature $T_{\text {eff }}$ and the surface gravity $g$ ), which is approximately 0.3-0.4 for CoRoT-2a (Claret 2000).

The "narrow-band hypothesis", i.e., a higher spot coverage in the planet-eclipsed section compared to the noneclipsed surface, also provides a natural explanation for the fact that both the eclipsed and noneclipsed surface regions account for about the same amplitude of variation in the lightcurve. Using the Sun as an analogy again, we would qualitatively expect the same structure, as seen under the planet path, on the opposite hemisphere as well: two "active belts" that are symmetric with respect to the equator. The noneclipsed activity belt, which would be only observable in the global lightcurve, would then be primarily responsible for the variability of the lightcurve contributed by the noneclipsed surface section.
We conclude that our results support a surface model consisting of active regions north and south of the equator, possibly even bands of spots at low latitudes analogous to the Sun. Further investigations of this system using more sophisticated models (first of all surface evolution) and using the entire observation interval of approximately 140 days have the potential of revealing more information on the constantly changing surface distribution of spots on CoRoT-2a.

Acknowledgements. K.H. is a member of the DFG Graduiertenkolleg 1351 Extrasolar Planets and their Host Stars. S.C. and U.W. acknowledge DLR support (50OR0105).

\section{References}

Alonso, R., Auvergne, M., Baglin, A., et al. 2008, A\&A, 482, L21 Alonso, R., Guillot, T., Mazeh, T., et al. 2009, A\&A, 501, L23

Auvergne, M., Bodin, P., Boisnard, L., et al. 2009, A\&A, 506, 411

Barnes, J. R., Cameron, A. C., Donati, J.-F., et al. 2005, MNRAS, 357, L1

Bouchy, F., Queloz, D., Deleuil, M., et al. 2008, A\&A, 482, L25

Claret, A. 2000, A\&A, 359, 289

Czesla, S., Huber, K. F., Wolter, U., Schröter, S., \& Schmitt, J. H. M. M. 2009, A\&A, accepted

Jeffers, S. V., Barnes, J. R., Cameron, A. C., \& Donati, J.-F. 2006, MNRAS, 366, 667

Lanza, A. F., Pagano, I., Leto, G., et al. 2009, A\&A, 493, 193

Press, W. H., Teukolsky, S. A., Vetterling, W. T., \& Flannery, B. P. 1992, Numerical recipes in C. The art of scientific computing, ed. W. H. Press, S. A. Teukolsky, W. T. Vetterling, \& B. P. Flannery (Cambridge University Press) Skrutskie, M. F., Cutri, R. M., Stiening, R., et al. 2006, AJ, 131, 1163

Vogt, S. S., \& Penrod, G. D. 1983, PASP, 95, 565

Wolter, U., Schmitt, J. H. M. M., Huber, K. F., et al. 2009, A\&A, 504, 561 